\title{
Video Distortion Using Region based DT-CWT Fusion
}

\author{
V. Supraja ${ }^{1}$, Y. Yashaswini², Vajjala Sravani², Valluru Sreevani², Poll Sharada Reddy ${ }^{1,2}$ \\ ${ }^{1}$ Associate Professor, Department of ECE, Ravindra College of Engineering for Women, Kurnool, Andhra \\ Pradesh, India
}

${ }^{2}$ Students, Department of ECE, Ravindra College of Engineering for Women, Kurnool, Andhra Pradesh, India. *Corresponding Author: sharadareddypoll@gmail.com

Article Info

Volume 8, Issue 4

Page Number : $313-321$

Publication Issue :

July-August-2021

\section{Article History}

Accepted : 01 Aug 2021

Published: 07 Aug 2021

\section{ABSTRACT}

Restoring a scene distorted by a region turbulence could be a difficult drawback in video police work. An image registration allows the geometric alignment of 2 pictures and is wide utilized in varied applications within the fields of remote sensing, a medical imaging and laptop vision. During this paper, we tend to propose a unique methodology for mitigating the consequences of a region distortion on discovered pictures. a region of an interest (ROI) for every frame is taken, to extract correct detail regarding objects behind the distorting layer. An easy and economical frame choice methodology is planned to pick informative ROIs, solely from smart quality frames. Every ROI ought to be register to cut back the distortion. The house variable drawback will be solved by image fusion mistreatment complicated ripple remodel. Finally distinction sweetening is applied.

Keywords : Dual Tree Complex Wavelet Transform (DT-CWT), Image Fusion, Region of Interest (ROI).

\section{INTRODUCTION}

Image fusion is that the technique of merging many pictures from multi-modal sources with several complementary info to create a brand-new image, that carries all the common also as complementary options of individual pictures. Numerous sorts of atmospherical distortion will influence the visual quality of video signals throughout acquisition. Supported temperature variations to scale back the distinction and atmospherical turbulence, thanks to distortions embody fog or haze. Once the temperature distinction between the bottom and also the air will increase then the thickness of every layer decreases, in robust turbulence, not solely scintillation, that produces small-scale intensity fluctuations within the scene and blurring effects square measure Present within the video imaging, however conjointly a cutting result happens and is perceived as completely different completely different components of objects occupancy different directions. Instances a system victimisation image fusion in any respect levels of process. This general structure can be used as a basis for any image process system. 
A. Single detector Image Fusion System

The basic single detector image fusion theme as shown in figure1 has been bestowed. The detector shown can be visible-band sensors or some matching band sensors. This detector captures the important world as a sequence of pictures. The sequence of pictures square measure then amalgamates along to come up with a brand-new image with optimum info content. As an example in illumination variant and abuzz atmosphere, somebody's operator might not be ready to find objects of his interest which may be highlighted within the resultant amalgamate image.

The fundamental single detector image combination arranges has been introduced. The detector indicated can be clear band sensors or some coordinative band sensors. This detector catches this gift reality as a briefing of images the processed camera is appropriate for daylight scenes; the infrared camera is correct in inadequately lit up things.

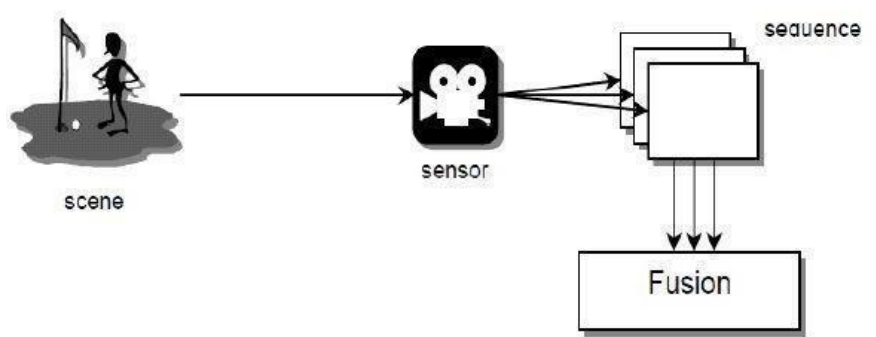

Fig 1 : Single sensor Image Fusion System

\section{B. Multi-Sensor Image Fusion System}

A multi-sensor image fusion theme overcomes the constraints of one sensing an element image fusion by merging the photographs from many sensors to create a composite image. Figure 2, illustrates a multi-sensor image fusion system. Here, associate degree infrared camera is related to the camera and their individual pictures are integrated to get a consolidated image. This approach overcomes the problems have observed before. The camera is appropriate for daylight scenes; the infrared camera is suitable in poorly lit environments.

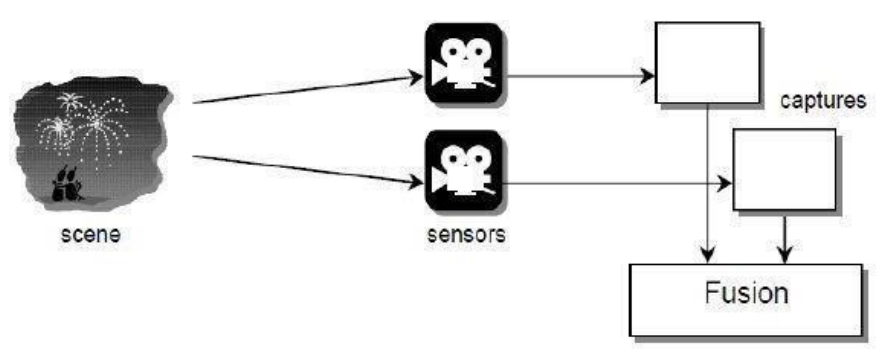

Fig 2 : Multi sensor Image Fusion System

\section{EXISTING SYSTEM}

Using varied ways, there has been vital analysis activity making an attempt to reliably reconstruct this convenient data. In observe, the right resolution is but not possible. Effective mitigation of part turbulence could be a difficult drawback. Modelprimarily based solutions ar impractical and blind deconvolution ways suffer from abstraction and temporal variation because of foreign terrorist organization. For large distortion and are timeoverwhelming, typical registration ways are ineffective. Finally, typical fusion ways need an outsized range of frames to pick out lucky regions. During this paper we have a tendency to introduce a brand-new approach that overcomes these issues. Image registration and Image fusion is performed by the twin Tree complicated riffle remodel (DT-CWT) domain since this provides close to shift-invariance and sensible property.

\section{A. Blind Deconvolution}

Blind deconvolution could be a deconvolution technique that allows recovery of the target scene from one or set of "blurred" pictures within the presence of a poorly determined or unknown purpose unfold operate (PSF). Regular linear and non- linear deconvolution techniques utilize a proverbial foreign terrorist organization. For blind deconvolution, the foreign terrorist organization is calculable from the image or image set, permitting the deconvolution to 
be performed. Researchers are learning blind deconvolution ways for several decades, and have approached the matter from completely different directions. Blind deconvolution may be performed iteratively, whereby every iteration improves the estimation of the foreign terrorist organization and also the scene, or non- iteratively, wherever one application of the formula, supported exterior data, extracts the foreign terrorist organization. Unvarying ways embrace most a posteriori estimation and expectation- maximization algorithms. A decent estimate of the foreign terrorist organization is useful for faster convergence however not necessary.

\section{B. Model primarily based process}

Model-based image process could be an assortment of techniques that have emerged over the past few decades that offer a scientific framework for the answer of inverse issues that arise in imaging applications. A physical system of some sort provides measurements, $Y$, that rely upon AN unknown signal or image, $X$. the target is then to work out the unknown signal or image, $X$, from these measurements. Since $X$ isn't directly determined, this drawback of decisive $\mathrm{X}$ from $\mathrm{Y}$ is thought as $\mathrm{AN}$ inverse drawback as a result of it needs that the physical method that generated the measurements be inverted or reversed to form $\mathrm{X}$ from $\mathrm{Y}$.

\section{Blind Deconvolution}

Blind deconvolution could be a deconvolution technique that allows recovery of the target scene from one or set of "blurred" pictures within the presence of a poorly determined or unknown purpose unfold operate (PSF). Regular linear and non- linear deconvolution techniques utilize a proverbial foreign terrorist organization. For blind deconvolution, the foreign terrorist organization is calculable from the image or image set, permitting the deconvolution to be performed. Researchers are learning blind deconvolution ways for many decades, and have approached the matter from completely different directions. Blind deconvolution may be performed iteratively, whereby every iteration improves the estimation of the foreign terrorist organization and also the scene, or non- iteratively, wherever one application of the formula, supported exterior data, extracts the foreign terrorist organization. Unvarying ways embrace most a posteriori estimation and expectation maximization algorithms. A decent estimate of the foreign terrorist organization is useful for faster convergence however not necessary.

\section{Model primarily based process}

Model-based image process could be an assortment of techniques that have emerged over the past few decades that offer a scientific framework for the answer of inverse issues that arise in imaging applications. A physical system of some sort provides measurements, $\mathrm{Y}$, that rely upon AN unknown signal or image, $X$. the target is then to work out the unknown signal or image, $X$, from these measurements. Since $\mathrm{X}$ isn't directly determined, this drawback of decisive $\mathrm{X}$ from $\mathrm{Y}$ is thought as $\mathrm{AN}$ inverse drawback as a result of it needs that the physical method that generated the measurements be inverted or reversed to form $\mathrm{X}$ from $\mathrm{Y}$.

\section{PROPOSED METHOD}

We propose another combination technique for decreasing the impacts of atmospherical turbulence as pictured. Some time recently taking the image combination we have a tendency to are taking ROI from the sides and arrangement them. At that time define determination is finished by the sharpness, force similitude and ROI size. Non-rigid image enrollment is connected. We have a tendency to then utilize a venue primarily based conceive to perform combination at the part level. This has focal points over pixel- primarily based getting ready since a lot of perceptive linguistics combination pointers will be thought of visible of real elements within the image, instead of on single or self- assertive gatherings of 
pixels. The mixture is performed within the twin Tree advanced moving ridge remodel (DT- CWT) that utilizes 2 various real separate moving ridge changes (DWT) to present the real and nonexistent elements of the CWT. 2 utterly blasted trees ar created, one for the odd specimens and one for the so, even specimens created at the principal level. This expansionsdirectional property over the DWT and might acknowledge positive and negative introductions giving six specific sub- groups at each level, comparison to $\pm 15^{\circ}, \pm 45^{\circ}, \pm 75^{\circ}$. Also, the amount of a DTCWT constant is powerful to commotion and worldly force varieties during this approach giving an efficient instrument to uprooting contorting swells. At long last, the DT-CWT is close to shift invarianta vital property for this application. Once combination, the impact of fog is cut utilizing regionally adjustive bar graph feat Finally distinction restricted adjustive bar graph feat is applied.

\section{A. ROI Alignment}

When mistreatment high magnification lenses, Capturing video within the presence of atmospherical turbulence, might cause the ROI in every frame to become misaligned. The ROI (orROIs) is manually marked within the initial frame. to seek out associate degree otsu threshold, the bar graph generated from the chosen ROI and therefore the encompassing space. Otsu threshold is employed to convert the image to a binary map. Associate degree erosion method is then applied. The aras connected to the sting of the subimage are removed, and therefore the step is performed iteratively till the realm close to the ROI is isolated.

The Block Diagram as shown in figure 3.

\section{Distorted Video}

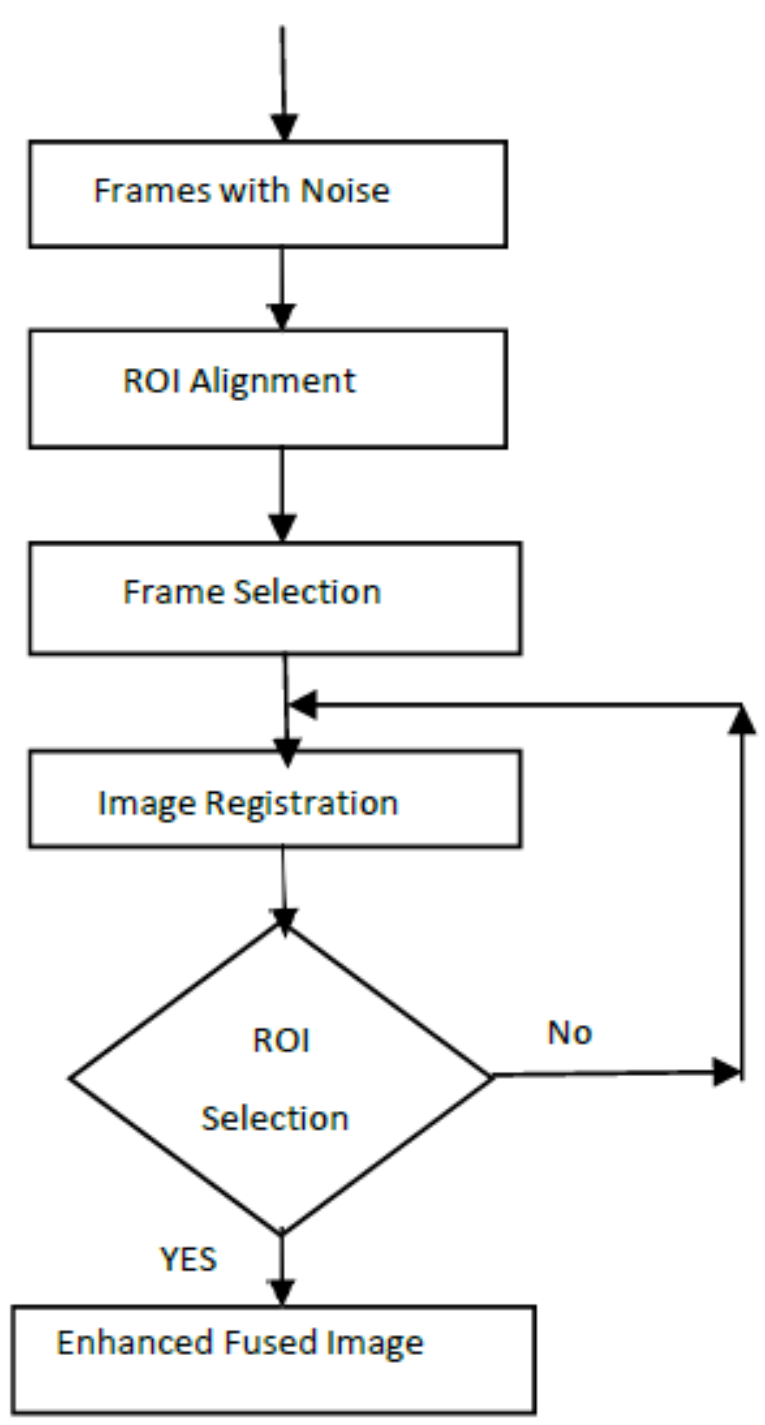

Fig 3: Block Diagram of Proposed Method

The same range of iterations is utilized in different frames with constant Otsu threshold. If there's over one isolated space, the world nighest in size and placement to the ROI within the 1st frame is employed. Finally, the centre of the mask in every frame is used to shift the ROI and align it across the set of frames. Note that the frames with incorrectly detected ROIs are removed within the frame choice method.

\section{B. Frame choice}

All frames within the sequence aren't wont to restore the image since the caliber frames may degrade the 
coalesced result. A set of pictures is fastidiously selected exploitation 3 factors: sharpness, intensity similarity and detected ROI size

1) Sharpness: $\mathrm{Gn}$ is one among the foremost necessary image quality factors and it will verify the number of detail a picture will convey. Here, the sharpness parameter $\mathrm{Gn}$ is computed from the summation of the high pass constant magnitudes.

2) Intensity Similarity: atomic number 50 is utilized to get rid of outliers. the foremost frames within the sequence contain fairly similar areas below assumption. Frames with considerably totally different content to others area unit probably to be greatly distorted. For scheming the mean sq. error (MSE), take the typical frame of the total sequence as a reference for frame $\mathrm{n}$. Then inverse MSE represents the similarity of every frame. It ought to be noted that this approach isn't strong to illumination changes.

3) Detected ROI Size: associate degree is that the total range of pixels contained within the ROI. ROIs area unit used as a result of it contains a lot of helpful data. the value perform $\mathrm{Cn}$ for frame $\mathrm{n}$ is computed exploitation.

$C_{n}=\frac{w_{G} G_{n}}{\lambda_{G}+\left|G_{n}\right|}+\frac{w_{S} S_{n}}{\lambda_{S}+\left|S_{n}\right|}+\frac{w_{A} A_{n}}{\lambda_{A}+\left|A_{n}\right|}$

Where wk and $\lambda \mathrm{k}$ are the load and slope management of the factors severally. The sigmoid operate is employed here to forestall one issue dominating the others, e.g. an obstruction unit could cause considerably high values of sharpness, however this frame ought to most likely not be enclosed within the elite dataset. The $\lambda \mathrm{k}$ is about to equal the mean of issue $\mathrm{k}$ so at the norm, its price is 0.5 . The price $\mathrm{Cn}$ is hierarchal from high to low. The Otsu technique will then be applied to seek out what percentage frames ought to be enclosed within the elite set.

\section{Image Registration}

Image registration is that the method of overlaying 2 or additional pictures of a similar scene taken at totally different times, from totally different viewpoints, and/or by totally different sensors. It geometrically aligns 2 pictures the reference and perceived pictures. these variations between pictures ar introduced thanks to totally different imaging conditions. During this paper, Registration of nonrigid bodies victimisation DTCWT, as planned in [15], is utilized. This algorithmic rule is predicated on phase-based dimensional volume registration, that is powerful to noise and temporal intensity variations. Motion estimation is performed iteratively, foremost by victimisation coarser level complicated coefficients to see massive motion elements then by using finer level coefficients to refine the motion field. Image registration has applications in remote sensing (cartography updating), and laptop vision. In

Medical pictures, and it's additionally utilized in astrophotography to align pictures taken of area. Image registration is crucial a part of broad image creation.

\section{Image Fusion}

Image fusion may be a method by that 2 or additional pictures ar combined into one image holding the vital options from every of the initial pictures. Thanks to its shift invariables, orientation property and multiscale properties, the DT-CWT is wide utilized in image fusion wherever helpful info from variety of supply pictures are elite and combined into a brandnew image variety of region-based fusion schemes are planned. These at first remodel pre-registered pictures victimisation AN mister remodel. Regions representing image options are then extracted from the remodel coefficients.

A grey-level clump employing a generalized pyramid linking technique is employed for segmentation. The regions ar then coalesced supported an easy region property like average activity. These strategies don't take full advantage of the wealth of data which will 
be calculated for every region. The image fusion rule is applied and a live of the common energy of the wave coefficients in a very region is usually an honest live of the importance of a region. In a very straightforward activity live taking absolutely the price of the wave constant is employed.

$$
P\left(r_{n}^{\theta}\right)=\frac{1}{\left|r_{n}^{\theta}\right|} \sum_{\forall I,(x, y) \in r_{n}^{\theta}}\left|d_{n}^{\theta, l}(x, y)\right| .
$$

The air-turbulence situation differs from alternative image- fusion issues because the segmentation boundaries that separate heterogeneous regions vary considerably from frame to border (due to turbulence distortion). To supply the sharpest and most temporally consistent boundaries for every region, we have a tendency to use the most of DT- CWT constant magnitudes over all frames rather than choosing only 1 region supported $\mathrm{P}(\mathrm{r} \theta \mathrm{n})$. To every boundary map $\mathrm{B} \theta, \mathrm{l}$ (constructed from the multistate watershed segmentation approach for every sub band $\theta$ at level 1), the dilation operation with a size of one element is applied. A second averaging filter is then applied to $\mathrm{B} \theta, \mathrm{l}$ to forestall separation when combining neighboring areas.The DT-CWT coefficients, $d \theta, 1$, of the amalgamated image may be written as

$$
\begin{aligned}
d^{\theta, l}= & \left(1-B^{\theta, l}\right) \sum_{R} \phi\left(d_{1}^{\theta, l}, d_{2}^{\theta, l}, \ldots, d_{N}^{\theta, l}\right) \\
& +B^{\theta, l} \max \left(d_{1}^{\theta, l}, d_{2}^{\theta, l}, \ldots, d_{N}^{\theta, l}\right) .
\end{aligned}
$$

The most essential dispute regarding image fusion is to make a decision a way to merge the sensing element pictures. In recent years, variety of image fusion ways are projected. One in all the primitive fusion schemes is pixel- by-pixel grey level average of the supply pictures.

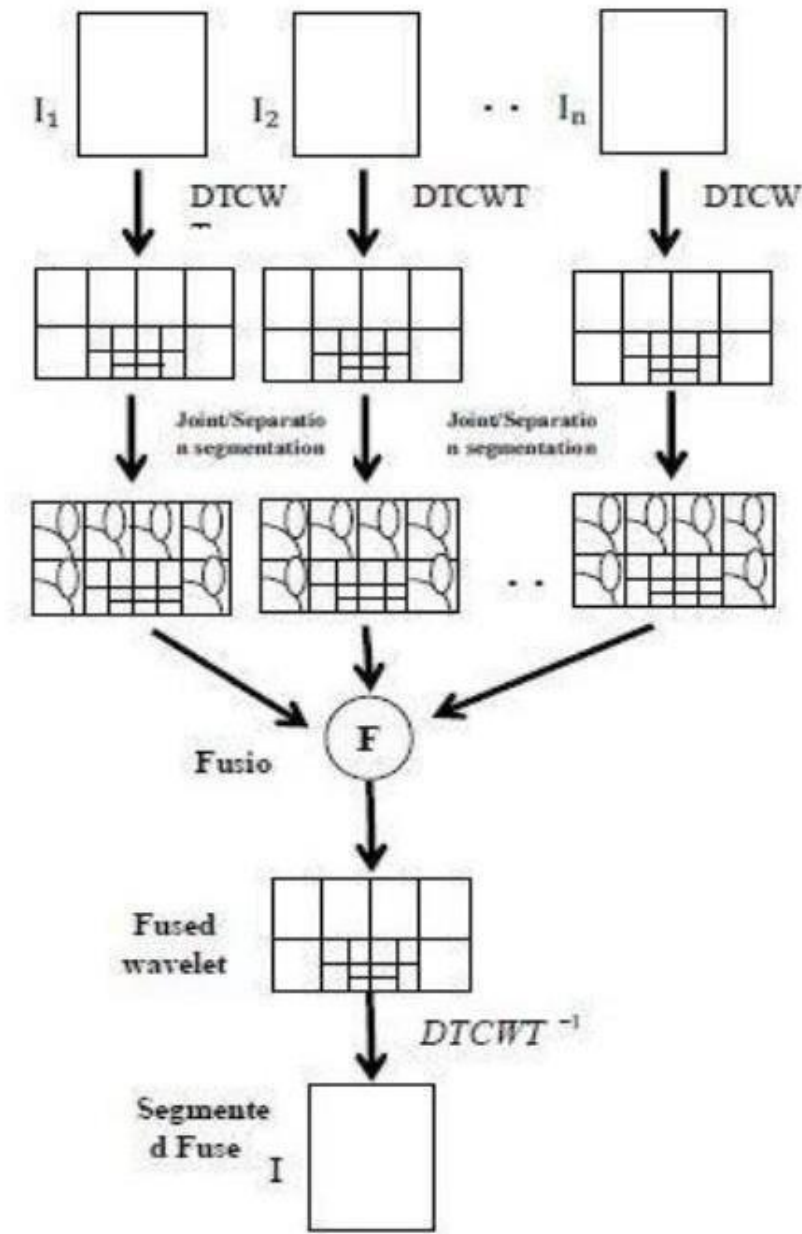

Fig 4: Region based image Fusion using DT-CWT

This simple technique in figure 4, usually has severe aspect effects like dropping the distinction. Some additional refined approaches began to develop with the launching of pyramid rework in mid-80s. Improved results were obtained with image fusion, performed within the rework domain. The pyramid rework solves this purpose within the reworked domain. The essential plan is to perform a multi resolution decomposition on every supply image, then integrate of these decomposition to develop a composite depiction.

\section{E. sweetening Techniques}

The principle objective of image sweetening is to method a given image so the result's additional appropriate than the first image for a particular application. It accentuates or sharpens image options like edges, boundaries, or distinction to form a 
graphic show additional useful for show and analysis.

The sweetening does not increase the inherent info content of the info, however it will increase the dynamic vary of the chosen options, so they will be detected simply

Contrast Enhancement: In several cases, atmospherically degraded pictures additionally suffer from poor distinction because of severe haze or fog. In such cases, pre- or post- processing is required to enhance image quality. Varied techniques are projected for haze reduction victimisation single pictures. Here we have a tendency to use a straightforward and quick technique using contrast restricted reconciling bar chart effort (CLAHE). The strategy enhances intensity regionally, therefore it's appropriate for applications that think about the ROI and its info content Quality Assessment: Image quality assessment is employed to live perceived image degradation, generally compared to a \{perfect or perfect image. This is often vital once assessing the performance of individual systems or for comparison totally different solutions. Image quality metrics are often classified in keeping with the provision of a reference (distortion-free) image, with that the distorted image is to be compared. Most existing approaches are classed as full-reference (FR), which means that a whole reference image is out there. Example francium ways embody Peak Signal to Noise quantitative relation (PSNR), period Structural Similarity (MSSSIM) sign to Noise quantitative relation (VSNR) and Perception-based Image Model (PIM).

These metrics ar utilized for evaluating the performance of the projected technique once a reference isn't on the market, as is usually the case for warmth haze reduction, quality assessment becomes difficult, and is spoken as no-reference (NR) or blind quality assessment. This is often delineated within the following section.

\section{EXPERIMENTAL RESULTS}

From Distorted video we are getting frames with noise, ROI alignment has been done to the frames later frames are selected. After retrieving the ROI from each frame, apply image registration using of non-rigid bodies using the phase shift properties of the DT-CWT.

The following are some of the pictures on figure 3,6 \&7 ROI, Target image, frame retrieved from a video, Fused image that are extracted from the project. This type of methods are mainly used to find a registration number plate of vehicle, to identify the persons in robbery case etc.

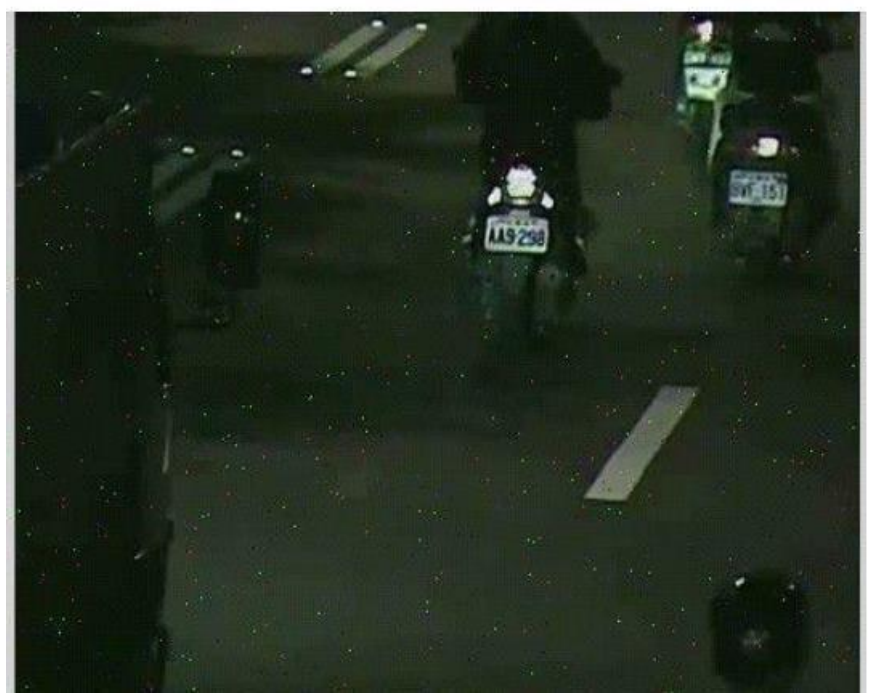

Fig 5: Frame retrieved from video

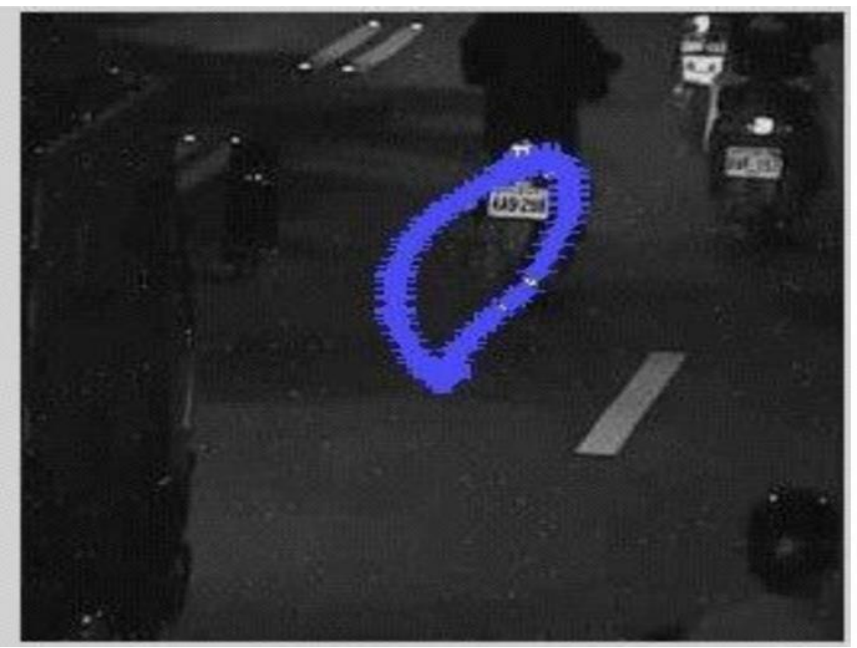

Fig 6: Region of Interest 


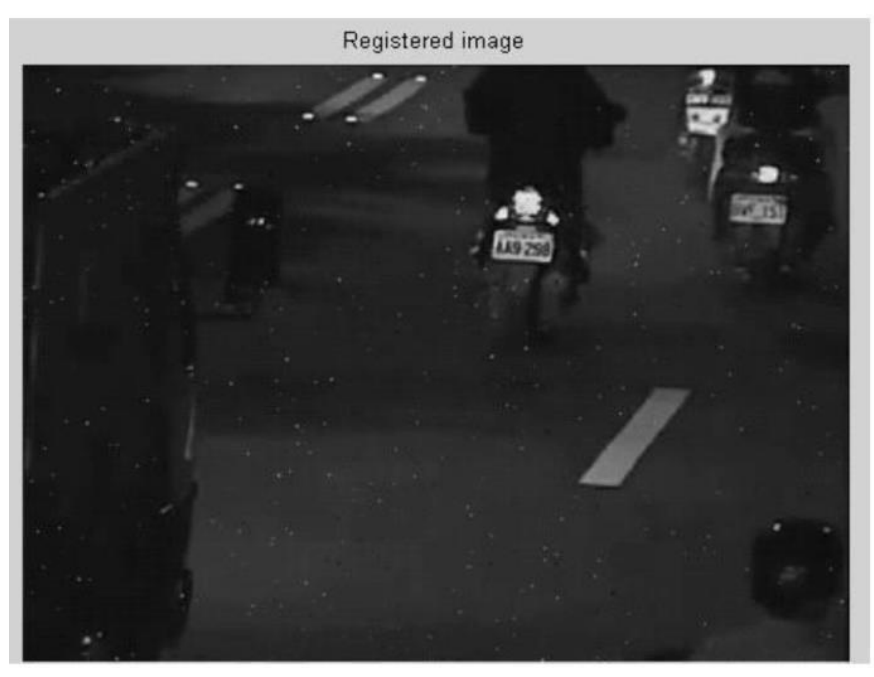

Fig 7: Registered image

\section{CONCLUSION\& FUTURE WORK}

This paper has introduced a replacement methodology for mitigating region distortion in longrange police investigation imaging. Vital enhancements in image quality square measure achieved exploitation region-based fusion within the DT-CWT domain. The price functions for frame choice to preprocess the distorted sequence. The method is completed with native distinction sweetening to cut back haze interference. From the distorted video we tend to get the standard of the one frame for the ROI image. In this paper, we've got given a distorted video as an input and take one frame as an output. In the future, distorted video is given as Associate in Nursing input Associate in Nursing take a multi frame as an output and build the frame into video. We will use the total frame for process the output. Whereas process the input image, 1st apply a frame choice exploitation sharpness of the image, intensity of the image and size of the image and calculate the price perform with the assistance of this parameter. We will do fusion for 2 pictures, one as a reference image and another because the input image and that we will fuse these 2 pictures to urge the multi-frame because the output. Finally, apply distinction sweetening to boost the standard of the image.

\section{REFERENCES}

[1]. L. C. Andrews, R. L. Phillips, C. Y. Hopen, and M. A. Al-Habash, "Theory of optical scintillation," J. Opt. Soc. Amer.A, vol. 16, no. 6, pp. 1417-1429, Jun. 1999.

[2]. H.S. Rana, "Toward generic military imaging adaptational optics," Proc. SPIE, vol. 7119, p. 711904, Sep. 2008.

[3]. B. Davey, R. Lane, and R. Bates, "Blind deconvolution of vociferous complex-valued image," Opt. Commun., vol. 69, nos.5-6, pp. 353356, 1989.

[4]. S. Harmeling, M. Hirsch, S. Sra, and B. Scholkopf, "Online blind image deconvolution for uranology," in Proc. IEEE Conf. CompPhotogr., Apr. 2009, pp. 1-7.

[5]. J. Gilles, T. Dagobert, and C. Franchis, "Atmospheric turbulence restoration by diffeomorphic image registration and blind deconvolution," in Proc. 10th Int. Conf. Adv.Concepts Intell. Vis. Syst., 2008, pp. 400409. 35, no. 1, pp. 157-170, Jan. 2013. Photography,Mar. 2010, pp. 1-8.

[6]. C. S. Huebner and C. Scheifling, "Software-based mitigation of image degradation because of atmospherical turbulence," Proc. SPIE, vol. 7828, pp.

[7]. N. Joshi and M. Cohen, "Seeing Mt. Rainier: Lucky imaging for multiimage denoising, sharpening, and haze removal," in Proc. IEEE Int. Conf. Comput. ol. 1. 2002, pp. 477-480. 78280N-1-78280N-12, Sep. 2010. 2005.

[8]. I. Selesnick, R. Baraniuk, and N. Kingsbury, "The dual- tree complicated moving ridge remodel," IEEE Signal method. Mag.,vol. 22, no. 6, pp. 123151, Nov.

[9]. Z. Wang, H. Sheikh, and A. Bovik, "Noreference sensory activity quality assessment of JPEG compressed pictures," in Proc.Int. Conf. Image method., v. 


\section{Cite this article as :}

V. Supraja, Y. Yashaswini, Vajjala Sravani, Valluru Sreevani, Poll Sharada Reddy, " Video Distortion Using Region based DT-CWT Fusion, International Journal of Scientific Research in Science, Engineering and Technology(IJSRSET), Print ISSN : 2395-1990, Online ISSN : 2394-4099, Volume 8, Issue 4, pp.313321, July-August-2021. Available at doi $\quad$ : https://doi.org/10.32628/IJSRSET218442

Journal URL : https://ijsrset.com/IJSRSET218442 